\title{
WANDERING DOMAINS FOR MAPS OF THE PUNCTURED PLANE
}

\author{
I. N. BAKER
}

\section{Introduction and results}

The iteration theory of Fatou [8,9] and Julia [12] applies to analytic maps $f: D \rightarrow D$ where the domain $D$ belongs to $\hat{\mathbf{C}}$, and introduces the sets $N(f)=$ $\left\{z ; z \in D,\left(f^{n}\right)\right.$ is a normal family in some neighbourhood of $\left.z\right\}$ and $J(f)=D \backslash N(f)$. To avoid trivial cases it is supposed that $f$ is not a Moebius transformation. The theory studies the way in which $J(f)$ divides the components of $N(f)$. To obtain interesting results it is necessary to assume that the complement of D consists of at most two points, since otherwise $J(f)$ is empty. We may assume that the complement of $D$ is $\emptyset,\{\infty\}$, or $\{0, \infty\}$ and with this normalisation there are essentially the following cases

I. $D=\hat{\mathbf{C}}, f$ rational,

II. $D=\mathbf{C}, f$ entire,

III. $D=\mathbf{C}^{*}=\{z ; 0<|z|<\infty\}$.

In the third case there are four types of function $f$, depending on the behaviour of $f$ at the isolated (potentially) singular points $0, \infty$,

(a) $f=k z^{n}, k \neq 0, n \in \mathbf{Z}, n \neq 0, \pm 1$

(N. B. we are excluding Moebius transformations),

(b) $f(z)=z^{n} \exp (g(z)), g$ non-constant entire, $n \in \mathbf{N}$,

(c) $f(z)=z^{-n} \exp (g(z)), g$ non-constant entire, $n \in \mathbf{N}$,

(d) $f(z)=z^{m} \exp \{g(z)+h(1 / z)\}, g, h$ non-constant entire functions, $m \in \mathbf{Z}$.

Here we have made the normalisation that if $f$ has exactly one essential singularity it is $\infty$. Note that (a), (b) may be regarded as belonging to cases I, II, and that for any $k \geqq 2$, and $f$ of type (c) we have $f^{k}$ of type (d).

The cases I, II have been discussed by Fatou [8, 9], Julia [12] and many other authors, case III by Rådström [14] and Bhattacharyya [6].

In all cases the set $J(f)$ is closed, non-empty and even perfect in $D$, with the invariance property $f(J(f))=f^{-1}(J(f))=J(f)$, (sometimes called "complete invariance") and the further one that $J\left(f^{p}\right)=J(f)$ for $p \in \mathbf{N}$. $N(f)$ may be empty, as is the case for $f(z)=\exp z$. 
If the components of $N(f)$ are denoted by $N_{j}$, then for each $N_{j}$ there is an $N_{k}$ such that $f\left(N_{j}\right) \subset N_{k} . N_{1}$ is a wandering component if $f^{m}\left(N_{1}\right) \cap f^{n}\left(N_{1}\right) \neq \emptyset, m, n \in \mathbf{N}$, implies that $m=n$. Sullivan [15] has shown that in case I there are no wandering components, while Baker [3, 4, 5], Eremenko and Lyubich [7], Herman [11] and Sullivan [15] have shown that wandering components may occur in case II. These wandering components may be either simply- or multiply-connected. In particular we have the following results.

Theorem A. [4] If $f$ is transcendental entire and $U$ is a multiply-connected component of $N(f)$, then $U$ is a wandering component. Further, $f^{n} \rightarrow \infty$ in $U(n \rightarrow \infty)$ and, for large $n, f^{n}(U)$ contains a closed curve $\gamma_{n}$, whose distance from 0 is arbitrarily large and whose winding number about 0 is non-zero. Moreover, every component of $N(f)$ is bounded.

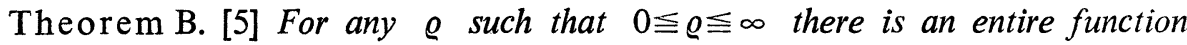
$f$ of order $\varrho$, which has multiply-connected wandering components of $N(f)$. In the case $\varrho=0$ the connectivity of the wandering component may be infinite.

If $U$ is one of the wandering components described in Theorem $\mathrm{B}$, it is clear that all $f^{n}(U),\left(n>n_{0}\right)$, are multiply-connected and that each is bounded away from 0 and $\infty$.

It is natural to ask whether wandering components can occur for functions of class III. It turns out that the situation described in Theorems A and B cannot occur but that simply-connected wandering domains are possible.

Theorem 1. If $f$ is a (non-Moebius) analytic map of $\mathbf{C}^{*}$ to itself, then the components of $N(f)$ are simply or doubly-connected. There is at most one doublyconnected component, except in the simple case $\operatorname{III}(a)$.

In case II, Theorem A states that any multiply-connected component of $N(f)$ is bounded. See also [2]. By contrast we have

Theorem 2. For $0<\alpha<1 / 2$ and $f(z)=\exp \left\{\alpha\left(z-z^{-1}\right)\right\}, N(f)$ consists of $a$ single multiply-connected component with 0 and $\infty$ on its boundary.

Theorem 3. There is a function $f$ of class III for which $N(f)$ has a doublyconnected component in which $f$ is analytically-conjugate to a rotation $z \rightarrow e^{i \alpha \pi} z$, $\alpha$ irrational.

This is the case of a Herman ring, which cannot occur for functions of class II.

The orem 4. There is a function of class III(b) which has a wandering component.

We remark that if $U$ is a wandering component for $f$ of class III, then $f^{n}(U)$ is simply-connected for $n \geqq n_{0}$. This will simplify attempts to prove that particular classes of such functions do in fact possess no wandering components, using the methods of [15], [4] or [7]. Some classes are already known from [4] and [7], e.g. $\exp (p(z))$, where $p$ is a polynomial. 


\section{Preliminary lemmas}

Suppose throughout that $f$ is one of the functions of class III(a), (b), (c) or (d).

Lemma 2.1. [6] Let $G$ be a component of $N(f)$ such that some sequence $f^{n_{k}}$, where $n_{k}$ is a strictly-increasing sequence of natural numbers, has a non-constant limit function $\varphi$ in $G$. Then for some $n_{k}$ we have $f^{n_{k}}(G)$ and $\varphi(G)$ contained in a component $G_{1}$ of $N(f)$, which is mapped univalently onto itself by some iterate $f^{p}$. Further the identity is a limit function of some sequence $f^{m_{k}}$ in $G_{1}$.

Lemma 2.2. [6] If $\alpha \in J(f)$ and $\Delta$ is a neighbourhood of $\alpha$ and $K$ is any compact subset of $\mathbf{C}^{*}$, then there is a natural number $n_{0}$ such that for $n>n_{0}$ we have $f^{n}(\Delta) \supset K$.

Lemma 2.3. [6] The fixed points of iterates of $f$ are dense in $J(f)$.

The following results were proved in the appropriate form for functions of class II in [1]. The proofs need almost no modification for class III.

Lemma 2.4. Suppose that $n_{k}$ is an increasing sequence of natural numbers such that certain branches $z=G_{n_{k}}(w)$ of the inverse functions of $w=f^{n_{k}}(z)$ are all defined and regular in the domain $G$. Then $\left(G_{n_{k}}\right)$ is a normal family in $G$.

Lemma 2.5. Let the set of singularities other than $0, \infty$ of $f^{-1}$ be $S$, and let $E$ be the set of points of the form $f^{n}(s), s \in S, n \geqq 0$. Then a point belongs to $E$ precisely if it is a singularity other than $0, \infty$ of some inverse function $f^{-n}$ of an iterate of $f$.

We may recollect that the singularities of $f^{-1}$ are either algebraic branch points or are asymptotic values approached by $f(z)$ as $z \rightarrow 0$ or $\infty$ along a suitable path.

Lemma 2.6. Let $E$ be the set defined in Lemma 2.5 and let $E^{\prime \prime}$ denote the derived set of $E$, together with any points which are of the form $f^{n}(s), s \in S$, for an infinity of values of $n$. Then any constant limit of a sequence $f^{n_{k}}$ in a component of $N(f)$ belongs to $L=E \cup E^{\prime \prime} \cup\{0, \infty\}=\bar{E} \cup\{0, \infty\}$.

Lemma 2.7. If the set $L$ defined in Lemma 2.6 has an empty interior and a connected complement, then no sequence ( $\left.f^{n_{k}}\right)$ has a non-constant limit function in any component of $N(f)$. 


\section{Proof of Theorem 1}

Lemma 3.1. Suppose that $f$ is of class III and that $G$ is a component of $N(f)$. Then if $\gamma$ is a simple closed curve in $G$, either (i) $\gamma$ separates $0, \infty$ or (ii) the complement of $\gamma$ has a compact component which belongs to $G$.

Proof. If (i) does not hold let $\Delta$ denote the compact component of $C^{*} \backslash \gamma$ and suppose that $\Delta \cap J(f) \neq \emptyset$. For an arbitrarily small positive $\varepsilon, f^{n}(\Delta), n>n_{0}(\varepsilon)$, covers $\mathbf{C}^{*}$ except for an $\varepsilon$-neighbourhood of 0 and $\infty$. Since $\partial\left(f^{n}(\Delta)\right) \subset f^{n}(\partial \Delta)=f^{n}(\gamma)$ it follows that $f^{n}(\gamma)$ meets the $\varepsilon$-neighbourhood of both 0 and $\infty$ for $n>n_{0}(\varepsilon)$. Thus if we pick out a subsequence $f^{n_{k}}$ which is locally uniformly convergent to, say, $\varphi$ in $G$, the function $\varphi$ is non-constant and (cf. Lemma 2.1) $\varphi(\gamma)$ is a compact subset of some component of $N(f) \subset \mathbf{C}^{*}$. This contradicts the fact that $d\left(f^{n}(\gamma), \infty\right) \rightarrow 0$ as $n \rightarrow \infty$.

Corollary. $G$ has at most two boundary components, so that the first part of Theorem 1 is proved.

Lemma 3.2. Suppose that $\gamma_{1}, \gamma_{2}$ are disjoint Jordan curves in $N(f), f$ of class III, which separate $0, \infty$. Then the region $\Delta$ bounded by $\gamma_{1}, \gamma_{2}$ contains no points of $J(f)$ except in the case when $f$ has the form III (a).

Proof. Suppose that $\Delta \cap J(f) \neq \emptyset$. Then for arbitrary positive $\varepsilon, f^{n}(\Delta)$ covers $C^{*}$ except for an $\varepsilon$-neighbourhood of 0 and $\infty$ for $n>n_{0}(\varepsilon)$. Now if some $f^{n_{k}}$ has a non-constant limit function $\varphi$ in the component $G_{1}$ of $N(f)$ which contains $\gamma_{1}$, it follows from Lemma 2.1 that for large $n, f^{n}\left(\gamma_{1}\right)$ is close to the compact set $\gamma_{1}^{\prime} \cup f\left(\gamma_{1}^{\prime}\right) \cup \ldots \cup f^{p-1}\left(\gamma_{1}^{\prime}\right) \subset \mathbf{C}^{*}, \gamma_{1}^{\prime}=\varphi\left(\gamma_{1}\right)$ where $p$ is the (smallest) positive integer such that $f^{p}$ maps to itself the component of $N(f)$ which contains $\varphi\left(G_{1}\right)$. By the covering property of $f^{n}(\Delta)$ it follows that for large $n, f^{n}\left(\gamma_{2}\right)$ contains points near both 0 and $\infty$, so that $f^{n}$ has no constant limit functions in the component $G_{2}$ of $N(f)$ which contains $\gamma_{2}$. But then $f^{n}\left(\gamma_{2}\right)$ must also approximate a certain compact subset of $\mathbf{C}^{*}$, as is the case for $f^{n}\left(\gamma_{1}\right)$. This again contradicts the covering property of $f^{n}(\Delta)$, as $n \rightarrow \infty$.

Thus for any sufficiently large $n$ we have either $\left|f^{n}\right|<\varepsilon$ on $\gamma_{1},\left|f^{n}\right|>\varepsilon^{-1}$ on $\gamma_{2}$ or $\left|f^{n}\right|>\varepsilon^{-1}$ on $\gamma_{1}$ and $\left|f^{n}\right|<\varepsilon$ on $\gamma_{1}$.

Thus the set $f^{n}\left(\gamma_{1}\right)$ or $f^{n}\left(\gamma_{2}\right)$ contains a simple closed curve $\gamma(\varepsilon)$ in $|z|>1 / \varepsilon$, which separates $0, \infty$. Further, on $\gamma(\varepsilon)$ we have either $|f|<\varepsilon$ or $|1 / f|<\varepsilon$. Applying this for $\varepsilon=1 / n \rightarrow 0$ and noting that for $m$ much larger than $n$ the curves $\gamma(1 / m), \gamma(1 / n)$ are disjoint, we see that either $f$ or $1 / f$ is bounded in a neighbourhood of $\infty$ and hence $f$ is analytic or has a pole at $\infty$. A similar argument applies at 0 . Thus $f$ has the form given by III(a).

Combining Lemma 3.2 with the corollary to Lemma 3.1 completes the proof of Theorem 1. 


\section{Proof of Theorem 2}

The function given by

$$
f(z)=\exp \left\{\alpha\left(z-z^{-1}\right)\right\}
$$

where $\alpha$ is a constant such that $0<2 \alpha<1$, is of type $\operatorname{III}(\mathrm{d})$. The unit circumference $\gamma$ is mapped so that $z=e^{i \vartheta}$ gives $f(z)=e^{i \varphi}$, where $\varphi=2 \alpha \sin \vartheta$, so that $|\varphi| \leqq 2 \alpha|\vartheta|$, and $f^{n}(z)=e^{i \vartheta_{n}}$, where $\left|\vartheta_{n}\right| \leqq(2 \alpha)^{n} \vartheta \rightarrow 0$. Thus $\gamma$ belongs to the domain of attraction of the attractive fixed point 1 for which $f(1)=1, f^{\prime}(1)=2 \alpha$.

The only singularities of $f^{-1}$ are 2 algebraic branch points, over $e^{2 \alpha i}$ and $e^{-2 \alpha i}$ respectively, and transcendental singularities over $0, \infty$. Denote by $G$ that component of $N(f)$ which contains 1 , and hence an annulus $A: 1-\delta<|z|<1+\delta$ for some $\delta>0$. Now we can reach all branches of $f^{-1}(1)$ by continuation from the value $f^{-1}(1)=1$ along paths in $A$. By the invariance properties of $N(f)$ it follows that all branches of $f^{-1}(1)$ belong to $G$. A similar argument shows that for any $z \in G$, all values of $f^{-1}(z)$ belong to $G$, by considering continuation of $f^{-1}$ from 1 to $z$ along a path in $G$.

Thus we have shown that $G$ is completely invariant. $G$ must therefore extend to the essential singularities 0 and $\infty$. It remains to show that there are no other components of $N(f)$.

Now in the notation of Lemma $2.6 \bar{E}=\left\{f^{n}\left(e^{ \pm 2 \alpha i}\right), n=0,1, \ldots,\right\} \cup\{1\}$, which is a compact subset of $G$, and $L=\bar{E} \cup\{0, \infty\}$. If $H$ is a component of $N(f)$ other than $G$, then the only possible limit functions of any subsequence of $f^{n}$ in $H$ are 0 and $\infty$. Thus $\left(f^{n}+f^{-n}\right) \rightarrow \infty$ in $H$ as $n \rightarrow \infty$. Since

$$
\left(f^{n}\right)^{\prime}(\tau)=f^{n}\left\{\prod_{v=1}^{n-1} \alpha\left(f^{v}+\frac{1}{f^{v}}\right)\right\} \alpha\left(1+z^{-2}\right)
$$

we see that if there is a sequence of $n$-values such that $f^{n} \rightarrow \infty$ in $H$, then for such $n$-values we also have $\left(f^{n}\right)^{\prime} \rightarrow \infty$ in $H$.

Put $g_{n}=\alpha\left(f^{n}-f^{-n}\right)$. Then for large $n$ in the given sequence we have $g_{n}^{\prime}$ large in $H$ and so, by Bloch's theorem, $g_{n}(H)$ contains a disc $\Delta$ of diameter at least $2 \pi$. Then $f^{n+1}(H) \supset \exp (\Delta)$ contains a circle $\gamma^{\prime}$ of the form $|z|=$ constant. By Lemma $3.2 \gamma^{\prime}$ belongs to $G$ and by the complete invariance of $G, H=G$ against assumption.

Thus if $H \neq G$ the only remaining possibility is that $f^{n} \rightarrow 0$ in $H$ as $n \rightarrow \infty$. Put $h_{n}=1 /\left(f^{n}\right)$, so that

$$
\left(h^{n}\right)^{\prime}(z)=-\left(f^{n}\right)^{-1}\left[\prod_{v=1}^{n-1}\left\{\alpha\left(f^{v}+\frac{1}{f^{v}}\right)\right\}\right] \alpha\left(1+z^{-2}\right) .
$$

Then $\left|\left(h^{n}\right)^{\prime}\right| \rightarrow \infty$ in $H$ and if $k_{n}=\alpha\left(h_{n}^{-1}-h_{n}\right)$, then $\left|k_{n}^{\prime}\right|=\left|\alpha\left(1+\left(f^{n}\right)^{2}\right) h_{n}^{\prime}\right| \rightarrow \infty$, so that $k_{n}(H)$ contains a disc $\Delta$ of diameter at least $2 \pi$. As above we then find that $f^{n+1}(H)=\exp \left(k_{n}(H)\right)$ contains a circumference $|z|=$ constant, and hence $H=G$. 
Remark. The map $z_{1}=f(z)$ is semiconjugated by $z=e^{i t}, z_{1}=e^{i t_{1}}$ to $t_{1}=2 \alpha \sin t=g(t)$, say. Our results give independent confirmation of the fact that $N(g)$ is a single simply-connected region in which $g^{n} \rightarrow 0$, a situation discussed e.g. by R. L. Devaney (unpublished).

\section{Proof of Theorem 3}

Modify the preceding example by setting

$$
g(z)=e^{2 \pi i \beta} z \exp \left\{\alpha\left(z-z^{-1}\right)\right\},
$$

where $\beta$ is a real constant. For $0<2 \alpha<1$ the function $g$ gives an orientation preserving homeomorphism of the unit circumference $\gamma$ to itself. Indeed, putting $e^{i \vartheta}=e^{2 \pi i x}$ on $\gamma$ we may represent $\left.g\right|_{\gamma}$ by $x \rightarrow G(x)=x+\beta+(\alpha / \pi) \sin (2 \pi x)(\bmod 1)$, which is monotone increasing and satisfies $G(x+1)=G(x)+1$. We recall the definition of the rotation number $\varrho$ of $g$

$$
\varrho=\lim _{n \rightarrow \infty} \frac{G^{n}(x)}{n}(\bmod 1),
$$

which is independent of $x$. $\varrho$ varies continuously with $g$ and $G$, in particular with $\beta$, and so $\beta$ may be chosen in such a way that $\varrho$ is an irrational number which satisfies a diophantine condition D.C.: - There exist $b \geqq 0, c>0$ such that for every $p / q \in \mathbf{Q}$ we have $|\varrho-(p / q)| \geqq c q^{-(2+\beta)}$. Such $\varrho$ are of measure 1 in $[0,1]$.

As was proved by Yoccoz [16], extending earlier work of Herman [10], $g$ is then real analytically conjugate on $\gamma$ to the rotation $z \rightarrow e^{2 \pi i i_{z}}$. The conjugacy is then in fact complex analytically valid in a neighbourhood $\Delta$ of $\gamma$, and $\Delta$ belongs to a component of $N(g)$ of the type whose existence was asserted in Theorem 3 . There are necessarily points of $J(g)$ near the essential singularities 0 and $\infty$ of $g$, so that the component is certainly multiply- and hence doubly-connected. This example is very closely related to the example $z \rightarrow z+(a / 2 \pi) \sin (2 \pi z)+b$ for case II, which was described in a slightly different context by Herman [11].

\section{Proof of Theorem 4}

Here we use a method of construction of wandering domains first introduced by A. Eremenko and M. Lyubich [7]. It is based on an approximation theorem.

If $F$ denotes a closed subset of $\mathbf{C}$ and $C_{a}(F)$ the functions which are continuous on $F$ and analytic in $\stackrel{\circ}{F}$, we say that $F$ is a Carleman set (for $\mathbf{C}$ ) if for any positive continuous functions $\varepsilon$ on $F$ and for any $g$ in $C_{a}(F)$ there is an entire $f$ such that $|g(z)-f(z)|<\varepsilon(z), z \in F$. By Arakelyan's theorem $\hat{\mathbf{C}} \backslash F$ must be connected and locally connected at $\infty$. A. H. Nersesjan [13] showed that if we add the following 
we have a sufficient condition for $F$ to be Carleman: for each compact $K$ the union $W(K)$ of those components of $\stackrel{\circ}{F}$ which meet $K$ is relatively compact (in $\mathbf{C}$ ).

It will follow that the set $F=B \cup \bigcup_{m=10}^{\infty}\left\{A_{m} \cup L_{m}\right\}$, is a Carleman set.

Now denote

$$
\begin{aligned}
L_{m} & =\{z ; \operatorname{Re} z=4 m\}, \quad m \geqq 10, \\
A_{m} & =\{z ;|z-4 m-2| \leqq 1\}, \quad m \geqq 10, \\
B & =\{z ;|z+6| \leqq 1\},
\end{aligned}
$$

and let $\delta, \delta_{m}^{\prime}$ be positive numbers so small that $|w-\pi i-\log 6|<\delta$ implies $\left|e^{w}+6\right|<$ $1 / 2$, and $|w-\log (4 m+2)|<\delta_{m}$ implies $\left|e^{w}-(4 m+2)\right|<1 / 2$. Using the approximation lemma we find an entire function $f$ such that

and

$$
\begin{aligned}
& |f(z)-\pi i-\log 6|<\delta, \quad z \in L_{m}, \quad m \geqq 10, \\
& |f(z)-\pi i-\log 6|<\delta, \quad z \in B,
\end{aligned}
$$

$$
|f(z)-\log (4 m+6)|<\delta_{m+1}, \quad z \in A_{m} \text {. }
$$

It follows that $g=e^{f}$, which is a function of class $\operatorname{III}(\mathrm{b})$, satisfies $g\left(A_{m}\right) \subset A_{m+1}$, so that $g^{n} \rightarrow \infty$ in each $A_{m}, m \geqq 10$. On the other hand $g$ maps $B$ into the smaller disc $|z+6|<1 / 2$, so that $B$ contains an attractive fixed point $\xi$ such that $g^{n} \rightarrow \xi$ in $B$, and $B \subset N(g)$.

Finally $g$ maps $L_{m}, m \geqq 10$, into $B$ so that $L_{m} \subset N(g)$ and further $L_{m}$ belongs to a component of $N(g)$ different from the component $G_{m}$ to which $A_{m}$ belongs. Thus each $G_{m}$ is a wandering component, mapping to $G_{m+1}$ under $z \rightarrow g(z)$.

\section{References}

[1] BAKER, I. N.: Limit functions and sets of non-normality in iteration theory. - Ann. Acad. Sci. Fenn. Ser. A I Math. 467, 1970, 1-11.

[2] BAKER, I. N.: The domains of normality of an entire function. - Ann. Acad. Sci. Fenn. Ser. A I Math. 1, 1975, 277-283.

[3] BAKER, I. N.: An entire function which has wandering domains. - J. Austral. Math. Soc. Ser. A 22, 1976, 173-176.

[4] BAKER, I. N.: Wandering domains in the iteration of entire functions. - Proc. London Math. Soc. (3) $49,1984,563-576$.

[5] BAKER, I. N.: Some entire functions with multiply-connected wandering domains. - Ergodic Theory and Dynamical Systems 5, 1985, 163-169.

[6] Bhattacharyya, P.: Iteration of analytic functions. - Ph. D. thesis, University of London, 1969 (unpublished).

[7] ERemenko, A., and M. Lyubich: Iterations of entire functions. - Dokl. Akad. Nauk SSSR 279, 1984, No. 1, 25-27 and preprint, Kharkov, 1984 (Russian).

[8] FAtou, P.: Sur les équations fonctionelles. - Bull. Soc. Math. France 47, 1919, 161-271 and $48,1920,33-94,208-314$.

[9] FATOU, P.: Sur l'itération des fonctions transcendantes entières. - Acta Math. 47, 1926, 337-370. 
[10] Herman, M. R.: Sur la conjugaison différentiable des difféomorphismes du cercle à des rotations. - Inst. Hautes Études Sci. Publ. Math. 49, 1979, 5-233.

[11] Herman, M. R.: Are there critical points on the boundary of singular domains? - Comm. Math. Phys. 99, 1985, 593-612.

[12] Julia, G.: Mémoire sur la permutabilité des fractions rationelles. - Ann. Sci. École Norm. Sup (3) 39, 1922, 131-215.

[13] Mergelyan, S. N.: Uniform approximation of functions of a complex variable. - Uspehi Mat. Nauk, 7, 1952, 31-122.

[14] RÅdSTRÖM, H.: On the iteration of analytic functions. - Math. Scand. 1, 1953, 85-92.

[15] Sullivan, D.: Quasiconformal homeomorphisms and dynamics I. - Ann. of Math. (2) 122, 1985, 401-418.

[16] Yoccoz, J. C.: Conjugaison differentiable des difféomorphismes du cercle dont le nombre de rotation vérifie une condition diophantienne. - Ann. Sci. École Norm. Sup. (4) 17, 1984, 333-359.

\author{
Imperial College \\ Department of Mathematics \\ London SW7 2BZ \\ England
}

Received 23 June 1986 